\title{
ASSESSING THE RESPONSE OF ESTUARINE INTERTIDAL ASSEMBLAGES TO URBANISED CATCHMENT DISCHARGE ${ }^{1}$
}

Glenn COURTENAY ${ }^{1,2}$, William GLADSTONE ${ }^{1}$ and Maria SCHREIDER ${ }^{1}$

1: Centre for Sustainable Use of Coasts and Catchments, School of Applied Sciences, University of Newcastle, (Ourimbah Campus), PO Box 127, Ourimbah, 2258 NSW Australia

2: Author for correspondence: Tel/fax: +61 24385 6632; email: courtenay@exemail.com.au

Abstract. Measurement of intertidal rock assemblages was investigated as a potential biological indicator to provide a quantitative estimate of the impact of urbanised catchment discharge on the estuaries of Sydney, Australia, from 1995 to 1999. Based on the presence and characteristics of adjacent human activities, sampling locations were categorised as: Bush; Urban; Urban with Sewer Overflows; and Industry with Sewer Overflows. In Sydney Harbour, variation in assemblage structure was measured between most impact categories, however differences between impact categories were not consistent for each year. Nevertheless, in years of above average rainfall (1998-99), reference assemblages adjacent to national parks and distant from urbanisation were different to all other putatively impacted assemblages. Variability within assemblages was least at reference locations in each year and greatest at locations adjacent to stormwater canals and sewer overflows, particularly in 19981999. Variation in assemblage structure in Sydney estuaries was most strongly correlated with chlorophyll-a concentrations. Univariate analysis also identified highly significant differences for a number of factors, however, interactions between year, impact categories and location for numerous analyses, confounded the differentiation between impact categories. The results suggest that intertidal rock assemblages in Sydney Harbour and surrounding estuaries appear to be responding to the quality and quantity of discharge from urbanised catchments and, furthermore, that assemblages are more suitable than individual taxa to indicate the difference between Bush and anthropogenically disturbed estuarine locations.

\footnotetext{
${ }^{1}$ This is the version of the manuscript that was accepted for publication in Environmental Monitoring and Assessment (2005) 107: 375-398.
} 
Keywords: anthropogenic impact, biological indicator, estuary, intertidal rock assemblage, urbanised catchments 


\section{Introduction}

Numerous biological indicators have been proposed to provide better understanding of how estuaries respond to environmental stresses over different temporal and spatial scales (Ward et al., 1998; Deeley and Paling, 1999). Much discussion has focussed on appropriate single indicator organisms for monitoring ecosystem change (Jones and Kaly, 1996). However, since exposure to a contaminant may change any of the distinct processes that govern the dynamics of an assemblage, monitoring of assemblages may provide a better assessment of impact or change (Maher and Norris, 1989). Recently, a number of Australian studies have used multivariate measures to detect disturbance effects as these methods are proving very sensitive for determining spatial and temporal variation in assemblage structure (Roberts et al., 1998; Kelaher et al., 1998; Glasby, 1999; Lindergarth and Hoskin, 2001).

Measurable changes in biota have been associated with sewage pollution in Australian estuaries. Sewage discharge enhances recruitment of mussels (Hindell and Quinn, 2000), alters the structure of soft bottom macrobenthic assemblages (Poore and Kudenov, 1978; Axelrad et al., 1979), degrades seagrass communities (Neverauskas, 1985) and encourages opportunistic epiphytes and benthic macroalgae (Neverauskas, 1985; Fairweather, 1990). Detection of impacts associated with sewage in estuarine environments, however, is typically confounded by the impacts of stormwater and other human activities (Raffaelli and Hawkins, 1999). Nevertheless, despite continued urbanisation along Australia's New South Wales (NSW) coast increasing the potential for human activities to impact on estuarine ecosystems, the effect of sewage-related contamination on estuarine intertidal rock assemblages remains largely undescribed.

Sydney Harbour, for example, is heavily urbanised and has, historically, received high pollutant loadings from stormwater runoff, sewer overflows and other diffuse sources during wet weather (AWT Ensight, 1997). Discharge of sewage effluent and stormwater impacts on 
the microbiological quality of the receiving waters and poses potential health problems and risks to aquatic life (Kueh and Grohmann, 1989; Kueh et al., 1991; Bickford et al., 1999).

Despite areas of fringing mangroves and urban national parks, the major catchments of the Sydney region (Port Jackson with its tributaries and Cooks River) largely incorporate residential housing, commercial and industrial development, and Sydney's three main sewerage transfer systems. In comparison, the catchments at the northern and southern extremities of the Sydney region (Pittwater and Port Hacking, respectively) have largely retained their natural state. These areas, however, are now also experiencing increased urbanisation.

Sydney's three main sewerage transfer systems are potential major pollution sources (AWT Ensight, 1997). Additionally, numerous overflow structures in the Sydney region may discharge into the waterways following a sewer choke or during power failure at a major pumping station or treatment plant, or as a result of stormwater infiltration into the sewerage system following moderate to heavy rainfall (AWT Ensight, 1997). In wet weather, urban stormwater also carries contaminants from a range of industrial and domestic sources, including chemicals contained in gardening products and vehicle exhaust particulate matter (Bickford et al., 1999). While undiluted sewage from overflows is toxic to sensitive aquatic species, Bickford et al. (1999) estimate that stormwater contributes approximately $85 \%$ of the total load of 'chemicals of concern to aquatic life' to Sydney’s coastal catchments.

In 1994, in response to a need for a system of measurement which would detect improvements in estuarine ecosystems resulting from Sydney Water Corporation's (SWC) capital works expenditure in the Sydney area, Australian Water Technologies (AWT) proposed the use of aquatic communities as indicators of the effectiveness of impact mitigation (Scammell and Collie, 1994). Between September 1995 and October 1999, five intertidal rock platform assemblage sampling events were conducted in the estuaries of the 
Sydney region to assess whether the composition of intertidal rock assemblages is related to anthropogenic disturbance in the form of urbanisation, industrialisation and the presence of sewer overflows, or the absence of such disturbance. Intertidal assemblages at locations in each of the impact categories were compared with assemblages at multiple reference locations. The objectives of the study were to test whether: (1) significant differences existed between the structure of intertidal rock assemblages at locations within impact categories for each year; (2) intertidal rock assemblages were significantly different between impact categories; (3) assemblages at impacted locations were more variable than at reference locations; and (4) significant differences existed between common indices of assemblage structure and abundance or coverage of individual taxa. Additionally, the study aimed to test whether the designation of locations to the impact categories was appropriate.

\section{Methods}

\section{$2.1 \quad$ STUDY LOCATIONS}

Prior to the commencement of sampling, results from previous water quality studies of Sydney estuarine locations were considered to define potentially useful impact categories and to select study locations (AWT Ensight, 1996). Impact categories were classified into: Urban; Urban with Sewer Overflows; and Industry with Sewer Overflows, based on the presence and characteristics of adjacent human activities.

Nineteen intertidal locations in the estuaries of the Sydney region were selected within Iron Cove, Lane Cove River, Cooks River, Port Jackson, Middle Harbour, Port Hacking and Pittwater (Figure 1; Table I). Each location was classified into an appropriate impact category based on available water quality data including levels of faecal coliforms, chlorophyll-a, total phosphorus, total nitrogen, oxidised nitrogen and total uncombined ammonia, relative to 
accepted guidelines (AWT Ensight, 1996, 1997; ANZECC, 2002). To negate the confounding influence of salinity from sewage-associated disturbances, only locations with a median salinity greater than $30 \%$ o (approximately $46 \mathrm{mS} . \mathrm{m}^{-1}$ conductivity, adjusted to $25^{\circ} \mathrm{C}$; UNESCO, 1980), calculated from available water quality data measured at $0.5 \mathrm{~m}$ depth, were included in the study. Reference locations were all adjacent to national parks where recorded concentrations of most variables were usually close to lower detection limits (AWT Ensight, 1996).

TABLE I Sydney estuarine locations where intertidal assemblages were sampled and impact
categories.

\begin{tabular}{llc}
\hline \multicolumn{1}{c}{ Waterway } & \multicolumn{1}{c}{ Location (Code) } & $\begin{array}{c}\text { Impact } \\
\text { Category }^{\#}\end{array}$ \\
\hline Port Jackson & Little Sirius Cove (L) & U \\
& Double Bay (J) & U \\
& Rushcutters Bay (Q) & US \\
& Blackwattle Bay (G) & IS \\
\hline Iron Cove & Hawthorne Canal* (H) & IS \\
& Iron Cove Creek* (I) & IS \\
\hline Middle Harbour & Davidson Park (B) & B \\
& Bantry Bay (A) & B \\
& Sugarloaf Bay (R) & US \\
& Tunks Park (S) & US \\
& Quakers Hat Bay (P) & US \\
\hline Pittwater & Towlers Bay (F) & B \\
& McCarrs Creek (D) & B \\
\hline Lane Cove River & Fig Tree Bridge (N) & US \\
\hline Cooks River & Cooks River (O) & US \\
\hline Port Hacking & Gunnamatta Bay (K) & U \\
& Maianbar (M) & U \\
\# Impact categories: Industry/Sewer (IS); Urban/Sewer (US); Urban (U); Bush (B) & B \\
* Sampling did not commence at this location until 1996. & B \\
\hline
\end{tabular}

While natural, intertidal, sandstone rocky reef was present at most locations, sandstone retaining walls were used at Hawthorne Canal and concrete bridge pylons were used at Fig Tree Bridge. Although variation in assemblages within a location can be attributed to differences in the complexity of the substratum (Minchinton, 1997; Beck, 1998; Glasby, 1999), Downes and Hindell (2000) found spatial variation to be more significant than 
substratum texture in accounting for large shifts in faunal composition at the family level. It was therefore assumed that any measurable variation in assemblages would be more attributable to differences in water quality rather than differences in rock surfaces.

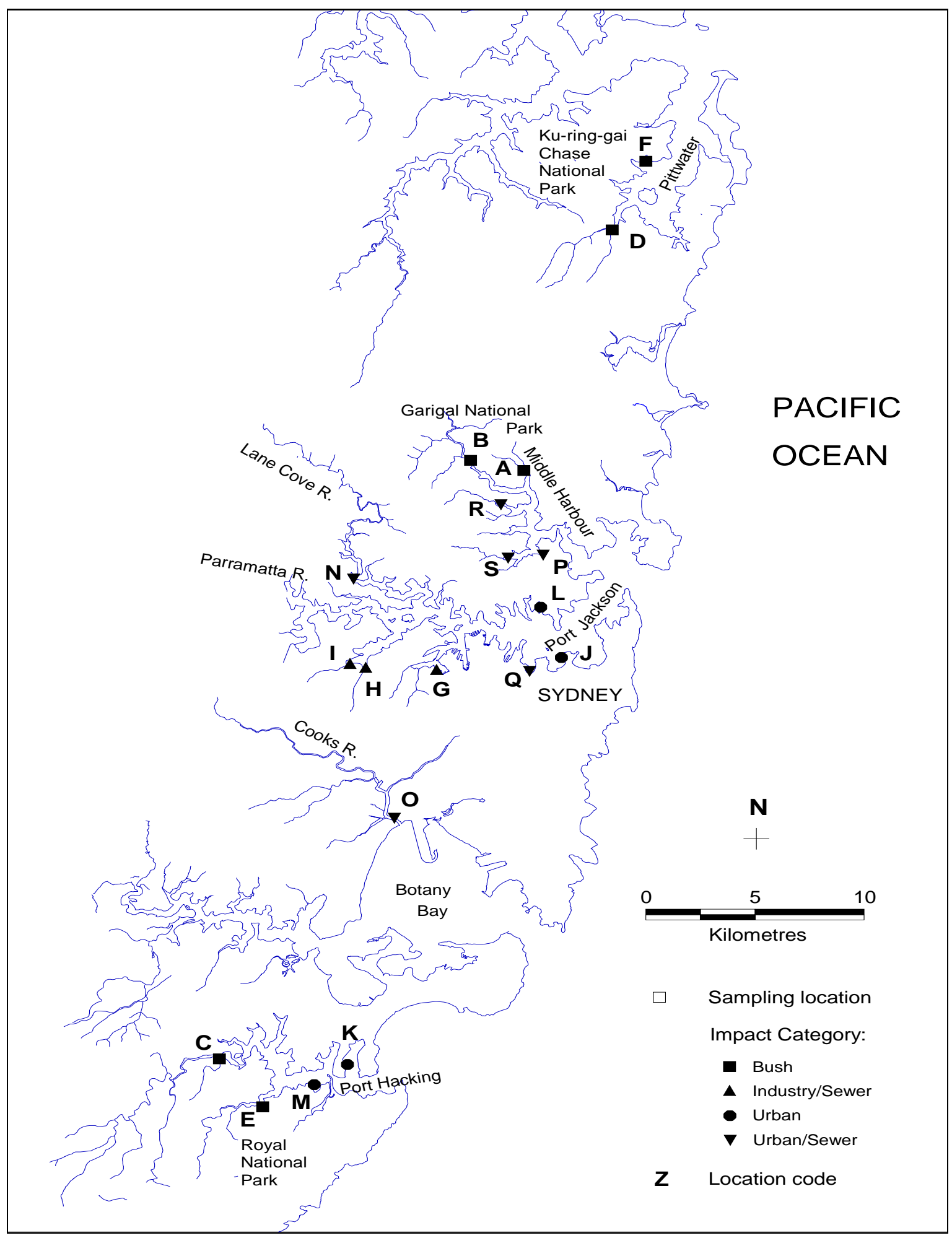

Figure 1. Sydney estuarine intertidal rock platform assemblage sampling locations. 


\subsection{FIELD SAMPLING}

Sampling was conducted within two hours before or after low tide and performed annually in September/October from 1995 to 1999. Measurements were recorded from 7 haphazardly placed $0.25 \mathrm{~m}^{2}$ quadrats on available rock or retaining wall surfaces at each location. The top of the quadrat was placed 10 to $15 \mathrm{~cm}$ below the upper growth line of Sydney Rock Oysters (Saccostrea glomerata) or the remains of the shells. Percentage cover of algae, encrusting bryozoans and polychaetes were estimated using grids of $0.01 \mathrm{~m}^{2}$ within the quadrat. Abundance (per quadrat) was recorded for all other taxa. Pilot studies indicated that 7 quadrats would provide adequate sampling of taxa at each location (AWT Ensight, 1996).

Organisms were identified to a range of recognisable taxonomic levels to allow for rapid assessment and minimal disturbance. Abundance data were aggregated to a relatively low level of taxonomic resolution as this generally results in no loss of information about patterns in assemblage structure (James et al., 1995; Vanderklift et al., 1996; Chapman, 1998; Thompson et al., 2003). Of the relatively abundant taxa, most molluscs and polychaetes were identified to genus, while barnacles were aggregated to class and green algae were identified to phylum. Less abundant taxa including Porifera, Cnidaria, Echinodermata, Platyhelminthes and Bryozoa were generally identified to phylum.

\subsection{WATER QUALITY DATA}

Data for total phosphorus, filterable phosphorus, total nitrogen, oxidised nitrogen, total uncombined ammonia, chlorophyll-a and faecal coliforms for each location were obtained from SWC and compared against accepted standards (ANZECC, 2002). Rainfall data collected at stations in the vicinity of the sampling locations were provided by the Bureau of Meteorology. The study commenced in 1994 after a period of prolonged dry weather, however most rainfall stations recorded above average rainfall for 1995 . While annual 
precipitation was generally below average through 1996 and 1997, above average rainfall was recorded at all stations in 1998 and 1999.

\section{$2.4 \quad$ STATISTICAL ANALYSES}

Multivariate and univariate analyses were initially conducted on data collected only from locations within Sydney Harbour and its tributaries (i.e. Bantry Bay, Davidson Park, Blackwattle Bay, Hawthorne Canal, Double Bay, Little Sirius Cove, Rushcutters Bay and Tunks Park). This choice of locations reduced any confounding influence of differences between estuaries. A balanced, orthogonal design of two locations nested within each of the four impact categories was used to test the null hypotheses that: (1) there were no significant differences between the structure of intertidal rock assemblages at locations within impact categories for each year; and (2) intertidal rock assemblages were not significantly different between impact categories. Additionally, we tested the hypothesis that assemblages at impacted locations are more variable than at reference locations (Warwick and Clarke, 1993). Univariate analyses tested the null hypotheses that there were no significant differences between: (1) common indices of assemblage structure (i.e. taxonomic richness, total abundance, heterogeneity, evenness); and (2) abundance or coverage of individual taxa. Analyses were only conducted for the years 1996-1999 as only one Industry/Sewer location was sampled in 1995.

\subsubsection{Multivariate Analysis}

Bray-Curtis similarity matrices, using fourth-root transformed data, were calculated for each year and non-metric Multidimensional Scaling (nMDS) ordinations were used to provide 
visual representations of sample similarities (Clark and Warwick, 2001). Analyses were conducted using PRIMER (V.5) software (Plymouth Marine Laboratories, UK).

The statistical significance of differences between assemblages from each of the a priori defined impact categories was tested in each year by two-way nested non-parametric multivariate analysis of variance (NPMANOVA; Anderson, 2001).

The similarity percentages (SIMPER) routine in PRIMER was used to determine the identity of taxa contributing to similarity within samples and dissimilarity between samples. To test the hypothesis that assemblage structure is more variable in impacted locations (Warwick and Clarke, 1993), differences in Bray-Curtis similarity values between replicates within each location were examined using the Multivariate Dispersion Indices (MVDISP) routine in PRIMER. MVDISP calculates the relative dispersion among replicates within each category, with larger values corresponding to greater within-category dispersion.

The BIOENV procedure in PRIMER was used to identify correlations between the observed patterns in intertidal assemblages and the following water quality data: mean, median and maximum values for each chemical variable and conductivity; minimum conductivity; geometric mean, median and maximum for faecal coliforms; and the total and maximum monthly rainfall. Most variables were recorded 12-15 times in the 12 months prior to intertidal sampling.

\subsubsection{Univariate Analysis}

Data were analysed using a 3-factor orthogonal model which examined differences between four fixed time periods (Years: 1996, 1997, 1998, 1999), four fixed impact categories (B, IS, U, US) and two random locations nested within each impact category. Prior to analysis of variance (ANOVA) using GMAV5 software (Institute of Marine Ecology, University of Sydney), Cochran's Test was used to test for homogeneity of variances and data were $\ln (\mathrm{x}+1)$ 
transformed to remove heterogeneity. Analysis was performed on untransformed data for variables which remained heterogeneous after transformation. Analysis of variance is relatively robust for most departures from homogeneous variances for balanced designs and sample sizes used in this study (Underwood, 1981, 1997).

While interactions between the factors were also measured, Underwood (1981) suggests that significant higher-order interactions reduce the scope for interpretation of differences between main effects. Student-Newman-Keuls (SNK) multiple comparison procedure was used to investigate patterns of differences. However, where significant interactions occurred, significant results for main effects were not reported.

\section{Results}

\subsection{ASSEMBLAGE STRUCTURE}

Twenty four taxa were identified in the Sydney estuaries during this study. Only 12 taxa were present at more than $20 \%$ of locations during any sampling occasion (Table II). Of these 12 taxa, 8 were not observed at a number of locations during any of the years sampled. Additionally, for each year, Austrocochlea spp., Hormosira banksii and chitons were not represented at the location where the minimum conductivity value was recorded in that year (i.e. Cooks River - 1995 and 1997, Fig Tree Bridge - 1996, Davidson Park - 1998, Tunks Park - 1999). Only Saccostrea glomerata, Bembicium spp., and green and red algae were recorded at all locations during the study. While no taxa were observed at all locations in each year, S. glomerata and green algae were observed at every location in 1997 and 1998, respectively. 
TABLE II

Taxa identified during the Sydney intertidal assemblage study. Taxa listed in the right-hand side column were identified at less than $20 \%$ of locations during any sampling occasion.

\begin{tabular}{|c|c|c|}
\hline TAXON & & TAXON \\
\hline \multirow[t]{8}{*}{ Molluscs } & & Molluscs \\
\hline & $\begin{array}{l}\text { Sydney Rock Oyster (Ostreidae) } \\
\text { Saccostrea glomerata }\end{array}$ & $\begin{array}{l}\text { Mudwhelks (Batillaridae) } \\
\text { Pyrazus sp., Batillaria sp. }\end{array}$ \\
\hline & $\begin{array}{l}\text { Mussel (Mytilidae) } \\
\text { Xenostrobus sp., Mytilus sp. }\end{array}$ & $\begin{array}{l}\text { Murex shell (Muricidae) } \\
\text { Morula sp. }\end{array}$ \\
\hline & $\begin{array}{l}\text { Periwinkle (Littorinidae) } \\
\text { Bembicium spp. }\end{array}$ & $\begin{array}{l}\text { Sea Slug } \\
\text { Onchidiidae/Aplysiidae }\end{array}$ \\
\hline & $\begin{array}{l}\text { Limpet (Acmaeidae) } \\
\text { Patelloida mimula }\end{array}$ & $\begin{array}{l}\text { Periwinkle (Littorinidae) } \\
\text { Nodilittorina unifasciata }\end{array}$ \\
\hline & $\begin{array}{l}\text { Other Limpets \& ‘false’ limpets } \\
\text { Patelloida spp., Cellana spp., } \\
\text { Siphonaria spp. }\end{array}$ & $\begin{array}{l}\text { Neritidae } \\
\text { Nerita atramentosa }\end{array}$ \\
\hline & $\begin{array}{l}\text { Top Shell (Trochidae) } \\
\text { Austrocochlea spp. }\end{array}$ & \\
\hline & $\begin{array}{l}\text { Chiton (Chitonidae) } \\
\text { Rhyssoplax spp. }\end{array}$ & \\
\hline \multirow[t]{4}{*}{ Algae } & & Algae \\
\hline & $\begin{array}{l}\text { Green Algae (Chlorophyta) } \\
\text { Enteromorpha spp., Ulva lactuca }\end{array}$ & \\
\hline & $\begin{array}{l}\text { Brown Algae (Phaeophyta) } \\
\text { Hormosira banksii }\end{array}$ & Other Brown Algae (Phaeophyta) \\
\hline & Red Algae (Rhodophyta) & $\begin{array}{l}\text { Coralline Algae (Corallinaceae) } \\
\text { Amphiroa sp. }\end{array}$ \\
\hline \multicolumn{2}{|c|}{ Crustaceans } & Echinoderms \\
\hline & $\begin{array}{l}\text { Barnacle (Cirripedia) } \\
\text { Balanus spp., Elminius spp., } \\
\text { Tesseropera rosea }\end{array}$ & Starfish (Asteroidea) \\
\hline \multicolumn{2}{|c|}{ Polychaetes } & Cnidaria \\
\hline & \multirow[t]{4}{*}{$\begin{array}{l}\text { Encrusting Polychaete } \\
\text { Galeolaria spp. }\end{array}$} & $\begin{array}{l}\text { Anemone (Anthozoa) } \\
\text { Actiniaria }\end{array}$ \\
\hline & & Porifera (sponges) \\
\hline & & Bryozoa \\
\hline & & Platyhelminths (flatworms) \\
\hline
\end{tabular}

nMDS ordinations showed a similar separation of impact categories in each year (Figure 2). Although overlap of categories did occur, nMDS ordinations placed Industry/Sewer locations apart from Bush locations on each occasion. Stress values were $\leq 0.15$ for each year suggesting little prospect of misinterpretation. 

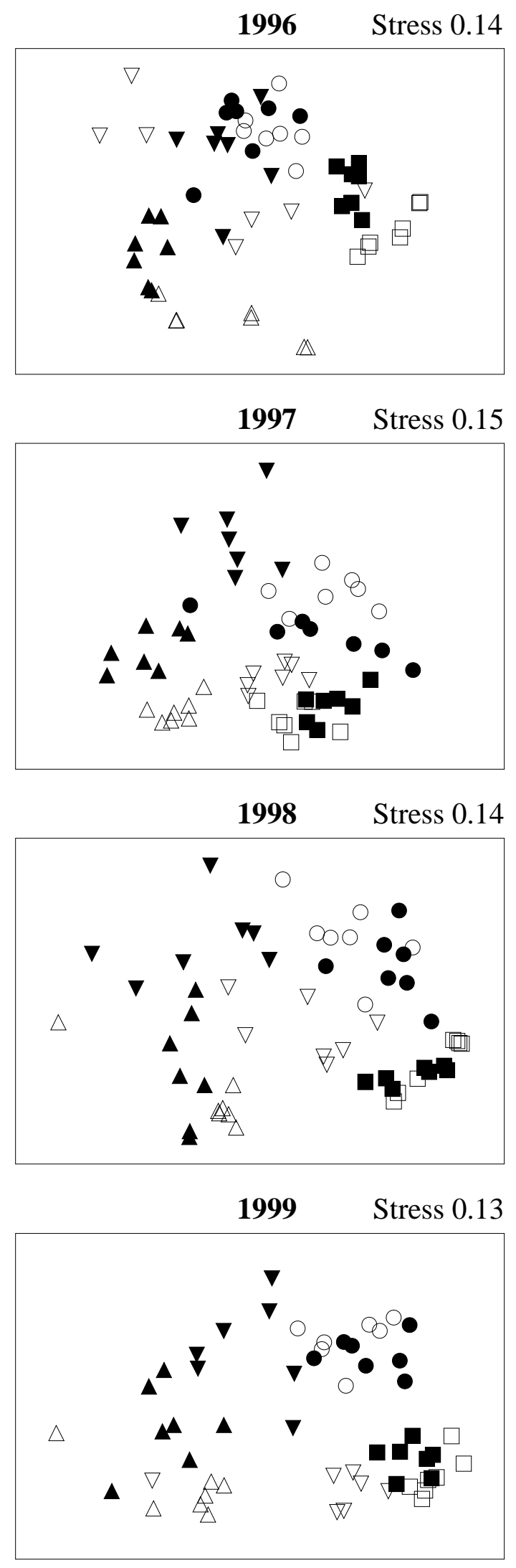

Figure 2. Non-metric multidimensional scaling (nMDS) ordinations comparing Sydney Harbour intertidal rock assemblages for two locations in each impact category for each year, i.e. Industry/Sewer $(\boldsymbol{\Delta} \Delta)$; Urban/Sewer $(\boldsymbol{\nabla} \nabla)$; Urban $(\bullet \circ)$; Reference/Bush $(\boldsymbol{\square})$. ( $n=7$ replicates per location). 
Two-way nested non-parametric multivariate analysis of variance identified consistent, significant differences between impact categories (Table III). Pair-wise a posteriori comparisons revealed that the Bush (B) category differed significantly from the Urban (U) category in each year, from the Industry/Sewer (IS) category in all years except 1996 and from the Urban/Sewer category (US) in 1998 and 1999 (Table III). The Urban category was significantly different to the Industry/Sewer category in all years but only to the Urban/Sewer category in 1998. Urban/Sewer and Industry/Sewer categories did not differ in any year.

Assemblages differed between locations in all impact categories (except Urban) in all years.

Relative dispersion values for the Bush locations were consistently below other impact categories (Table IV). In contrast, variability at the Urban/Sewer locations was greatest in all years sampled. The greatest range of dispersion values between the impact categories was measured in 1998 and 1999 when above average rainfall was experienced in the Sydney region.

Barnacles and green algae (Chlorophyta) contributed the greatest amount to assemblage similarity at the Industry/Sewer locations in all years. S. glomerata, P. mimula and Bembicium spp. contributed most to the similarity at Bush locations in all years (Table V). Green algae contributed relatively more to similarity at Urban/Sewer locations in years of above average rainfall (1998-1999). In the same years, the relative contributions of green algae decreased and Bembicium spp. increased at Bush locations. Furthermore, the relative contribution of Galeolaria sp. to similarity decreased in all impact categories in 1998-1999. The contribution of green algae and barnacles to assemblage similarity was generally lower at Urban locations while the contribution of oysters was generally higher. 
TABLE III

Results of two-way nested non-parametric multivariate analysis of variance (NPMANOVA) comparing impact categories and locations within each impact category in Sydney Harbour waterways in each year and results of pair-wise a posteriori tests between impact categories in each year.

\begin{tabular}{|c|c|c|c|c|c|c|c|c|}
\hline Year & Source of variation & $\mathrm{df}$ & MS & $F$ & $p$ & Impacts & $t$ & $p$ \\
\hline 1996 & $\begin{array}{l}\text { Impact } \\
\text { Location (Impact) } \\
\text { Residual }\end{array}$ & $\begin{array}{r}3 \\
4 \\
48\end{array}$ & $\begin{array}{r}21284.99 \\
4536.52 \\
534.87\end{array}$ & $\begin{array}{l}4.69 \\
8.48\end{array}$ & $\begin{array}{l}0.023 \\
0.001\end{array}$ & $\begin{array}{l}\text { Bush \& Industry/Sewer } \\
\text { Bush \& Urban } \\
\text { Bush \& Urban/Sewer } \\
\text { Industry/Sewer \& Urban } \\
\text { Industry/Sewer \& Urban/Sewer } \\
\text { Urban \& Urban/Sewer }\end{array}$ & $\begin{array}{l}2.52 \\
3.50 \\
2.28 \\
2.35 \\
1.44 \\
1.40\end{array}$ & $\begin{array}{l}0.052 \\
0.021 \\
0.083 \\
0.035 \\
0.219 \\
0.191\end{array}$ \\
\hline 1997 & $\begin{array}{l}\text { Impact } \\
\text { Location (Impact) } \\
\text { Residual }\end{array}$ & $\begin{array}{r}3 \\
4 \\
48\end{array}$ & $\begin{array}{r}15439.81 \\
3535.22 \\
515.92\end{array}$ & $\begin{array}{l}4.37 \\
6.85\end{array}$ & $\begin{array}{l}0.012 \\
0.001\end{array}$ & $\begin{array}{l}\text { Bush \& Industry/Sewer } \\
\text { Bush \& Urban } \\
\text { Bush \& Urban/Sewer } \\
\text { Industry/Sewer \& Urban } \\
\text { Industry/Sewer \& Urban/Sewer } \\
\text { Urban \& Urban/Sewer }\end{array}$ & $\begin{array}{l}3.35 \\
2.85 \\
1.55 \\
3.28 \\
1.56 \\
1.26 \\
\end{array}$ & $\begin{array}{l}0.022 \\
0.015 \\
0.154 \\
0.006 \\
0.138 \\
0.256 \\
\end{array}$ \\
\hline 1998 & $\begin{array}{l}\text { Impact } \\
\text { Location (Impact) } \\
\text { Residual }\end{array}$ & $\begin{array}{r}3 \\
4 \\
48\end{array}$ & $\begin{array}{r}20063.98 \\
2526.42 \\
543.02\end{array}$ & $\begin{array}{l}7.94 \\
4.65\end{array}$ & $\begin{array}{l}0.006 \\
0.001\end{array}$ & $\begin{array}{l}\text { Bush \& Industry/Sewer } \\
\text { Bush \& Urban } \\
\text { Bush \& Urban/Sewer } \\
\text { Industry/Sewer \& Urban } \\
\text { Industry/Sewer \& Urban/Sewer } \\
\text { Urban \& Urban/Sewer }\end{array}$ & $\begin{array}{l}4.26 \\
3.44 \\
2.65 \\
3.46 \\
1.70 \\
2.11\end{array}$ & $\begin{array}{l}0.014 \\
0.009 \\
0.021 \\
0.007 \\
0.113 \\
0.039\end{array}$ \\
\hline 1999 & $\begin{array}{l}\text { Impact } \\
\text { Location (Impact) } \\
\text { Residual }\end{array}$ & $\begin{array}{r}3 \\
4 \\
48\end{array}$ & $\begin{array}{r}15826.87 \\
1937.87 \\
413.12\end{array}$ & $\begin{array}{l}8.17 \\
4.69\end{array}$ & $\begin{array}{l}0.002 \\
0.001\end{array}$ & $\begin{array}{l}\text { Bush \& Industry/Sewer } \\
\text { Bush \& Urban } \\
\text { Bush \& Urban/Sewer } \\
\text { Industry/Sewer \& Urban } \\
\text { Industry/Sewer \& Urban/Sewer } \\
\text { Urban \& Urban/Sewer }\end{array}$ & $\begin{array}{l}5.29 \\
4.86 \\
2.04 \\
4.52 \\
1.69 \\
1.78 \\
\end{array}$ & $\begin{array}{l}0.009 \\
0.003 \\
0.047 \\
0.003 \\
0.099 \\
0.067\end{array}$ \\
\hline
\end{tabular}

TABLE IV

Relative dispersion of assemblages within impact categories within Sydney Harbour waterways for each year.

\begin{tabular}{lllll}
\hline Impact category & 1996 & 1997 & 1998 & 1999 \\
\hline Bush & 0.646 & 0.620 & 0.501 & 0.457 \\
Industry/Sewer & 1.190 & 0.854 & 1.034 & 1.167 \\
Urban & 0.811 & 1.254 & 1.044 & 0.888 \\
Urban/Sewer & 1.353 & 1.272 & 1.421 & 1.488 \\
\hline
\end{tabular}


TABLE V

Percentage contribution of each taxon to the similarity in assemblage structure between locations in each impact category within Sydney Harbour waterways for each year (to a cumulative percentage of at least 90\%).

\begin{tabular}{|c|c|c|c|c|c|c|c|c|c|c|c|c|c|}
\hline Year & $\begin{array}{l}\text { Impact } \\
\text { category }\end{array}$ & 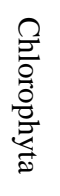 & 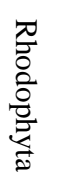 & 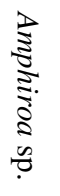 & $\begin{array}{l}0 \\
0 \\
0 \\
0 \\
\vdots \\
\vdots \\
0 \\
0 \\
0\end{array}$ & 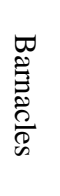 & 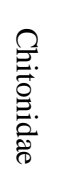 & 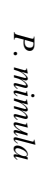 & 莺 & 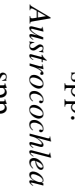 & : & 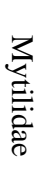 & 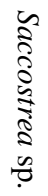 \\
\hline \multirow[t]{4}{*}{1996} & Industry/Sewer & 45 & & & & 46 & & & & & & & \\
\hline & Urban/Sewer & 12 & 8 & & 28 & 19 & & & 13 & & & & 11 \\
\hline & Urban & 3 & & & 14 & 17 & 11 & & 19 & 7 & & & 20 \\
\hline & Bush & 8 & & & & & & 30 & & & 15 & 6 & 36 \\
\hline \multirow[t]{4}{*}{1997} & Industry/Sewer & 30 & & & & 49 & & & & & 8 & 8 & \\
\hline & Urban/Sewer & 9 & & 6 & 9 & 12 & & & 20 & & 9 & 12 & 15 \\
\hline & Urban & & & & 9 & 9 & & & 10 & 13 & 15 & & 37 \\
\hline & Bush & 8 & & & & & & 25 & & & 19 & 12 & 30 \\
\hline \multirow[t]{4}{*}{1998} & Industry/Sewer & 30 & & & & 43 & & & & & 6 & 16 & \\
\hline & Urban/Sewer & 35 & 11 & & & & & 9 & 8 & & & 10 & 17 \\
\hline & Urban & & & & & 12 & & 6 & 17 & 13 & 14 & & 27 \\
\hline & Bush & & & & & & & 29 & & & 28 & & 37 \\
\hline \multirow[t]{4}{*}{1999} & Industry/Sewer & 25 & & & & 45 & & & & & 10 & 11 & \\
\hline & Urban/Sewer & 25 & & & & & & 4 & 9 & & 16 & 16 & 20 \\
\hline & Urban & & & & & 9 & 10 & 8 & 13 & 15 & 19 & & 20 \\
\hline & Bush & & & & & & & 25 & & & 30 & 10 & 33 \\
\hline
\end{tabular}

TABLE VI

Percentage contribution of each taxon to the dissimilarity in assemblage structure between impact categories within Sydney Harbour waterways for each year (to a cumulative percentage of at least 90\%).

\begin{tabular}{|c|c|c|c|c|c|c|c|c|c|c|c|c|c|}
\hline Year & $\begin{array}{l}\text { Impact } \\
\text { categories* }\end{array}$ & 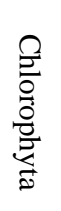 & 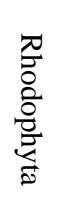 & 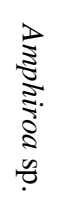 & $\begin{array}{l}0 \\
\frac{0}{0} \\
0 \\
\vdots \\
\vdots \\
0 \\
0 \\
0\end{array}$ & 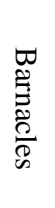 & 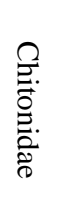 & $\begin{array}{l}0 \\
\vdots \\
\vdots \\
\vdots \\
\vdots \\
\vdots\end{array}$ & 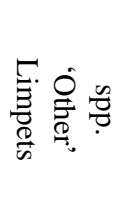 & 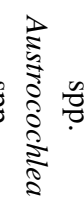 & 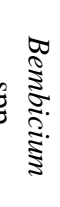 & 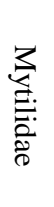 & 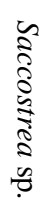 \\
\hline \multirow[t]{6}{*}{1996} & B - IS & 10 & & & & 26 & & 16 & 6 & & 8 & 9 & 18 \\
\hline & $\mathrm{B}-\mathrm{U}$ & 5 & & 5 & 12 & 15 & 9 & 13 & 11 & 7 & 7 & 6 & 4 \\
\hline & B - US & 7 & 7 & & 14 & 14 & & 15 & 9 & & 9 & 7 & 12 \\
\hline & IS - U & 11 & & 4 & 10 & 17 & 8 & & 13 & 7 & 5 & 6 & 13 \\
\hline & IS - US & 11 & 8 & & 15 & 24 & & & 10 & & 5 & 11 & 9 \\
\hline & U - US & 8 & 7 & 6 & 6 & 13 & 10 & 5 & 11 & 8 & 6 & 7 & 10 \\
\hline \multirow[t]{6}{*}{1997} & B - IS & 9 & & & & 26 & & 16 & 5 & & 7 & 11 & 15 \\
\hline & $\mathrm{B}-\mathrm{U}$ & 10 & 5 & & 9 & 12 & & 14 & 8 & 10 & 7 & 12 & 4 \\
\hline & B - US & 8 & 4 & 9 & 9 & 11 & 4 & 10 & 11 & & 8 & 8 & 8 \\
\hline & IS - U & 15 & & & 7 & 19 & & 4 & 7 & 9 & 7 & 10 & 15 \\
\hline & IS - US & 10 & & 8 & 8 & 18 & 4 & 6 & 11 & & 8 & 11 & 9 \\
\hline & U - US & 9 & & 9 & 7 & 9 & 5 & 7 & 9 & 10 & 9 & 11 & 9 \\
\hline \multirow[t]{6}{*}{1998} & B - IS & 12 & & & & 23 & & 18 & & 3 & 9 & 10 & 18 \\
\hline & B - U & 4 & 3 & & 6 & 12 & 5 & 15 & 16 & 10 & 7 & 5 & 6 \\
\hline & B - US & 15 & 8 & 4 & & 5 & & 14 & 8 & 4 & 14 & 7 & 14 \\
\hline & IS - U & 13 & & & 4 & 16 & 4 & 5 & 10 & 8 & 7 & 10 & 14 \\
\hline & IS - US & 5 & 8 & 5 & & 27 & & 8 & 8 & & 9 & 12 & 11 \\
\hline & U - US & 15 & 7 & 5 & 5 & 8 & & 6 & 9 & 10 & 9 & 7 & 9 \\
\hline \multirow[t]{6}{*}{1999} & B - IS & 13 & & & & 29 & & 16 & & & 11 & 9 & 15 \\
\hline & B - U & 6 & & & 6 & 13 & 12 & 11 & 15 & 13 & & 10 & 6 \\
\hline & B - US & 16 & 4 & & 4 & 7 & & 16 & 11 & 5 & 11 & 8 & 11 \\
\hline & IS - U & 10 & & & & 20 & 8 & 7 & 9 & 11 & 7 & 10 & 11 \\
\hline & IS - US & 6 & 4 & & 4 & 32 & & 6 & 9 & & 9 & 11 & 10 \\
\hline & U - US & 11 & & & 5 & 10 & 9 & 8 & 8 & 12 & 7 & 11 & 7 \\
\hline
\end{tabular}

* Industry/Sewer (IS); Urban (U); Urban/Sewer (US); Bush (B). 
Although the major taxa contributing to dissimilarity between impact categories varied between impact combinations, the large numbers of barnacles recorded at Industry/Sewer locations each year resulted in this taxa contributing the greatest amount to the dissimilarity between Industry/Sewer and all other impact categories in all years (Table VI). Green algae contributed most to dissimilarity between Bush and Urban/Sewer categories in the years which experienced above average rainfall (1998-1999).

\subsection{PATTERNS OF ABUNDANCE OF BIOTA}

Significant Year x Impact Category and/or Year x Location (Impact Category) interactions occurred for all variables except total abundance and abundance of barnacles and limpets (Table VII). Therefore, for most variables analysed, the main effects were not examined as the patterns of differences between impact categories varied significantly between years or the magnitude of the differences between locations within each impact category varied between years.

\subsubsection{Diversity Indices}

Differences in taxonomic richness between impact categories in Sydney Harbour varied between years as indicated by the significant Year x Impact interaction (Table VII). Lowest taxonomic richness occurred at an Industry/Sewer location in all years (Figure 3a). Highest taxonomic richness occurred at an Urban location in all years except 1997.

No significant differences in total abundance were measured between years or impact categories. However, SNK tests identified highly significant differences in total abundance between locations within the Industry/Sewer category (Table VII). Differences in total abundance between these locations was greatest (1996) and least (1997) in years when below 
TABLE VII

Summaries of results of three factor ANOVA comparing impact categories and locations within each impact category in Sydney Harbour waterways.

\begin{tabular}{|c|c|c|c|c|c|c|c|c|c|c|}
\hline \multirow[t]{2}{*}{ Source } & \multirow[t]{2}{*}{ df } & \multirow[t]{2}{*}{$F$-ratio vs } & \multicolumn{2}{|c|}{ Taxonomic Richness $^{\mathrm{a}}$} & \multicolumn{2}{|c|}{ Abundance $^{\mathrm{a}}$} & \multicolumn{2}{|c|}{ Heterogeneity $^{\mathrm{b}}$} & \multicolumn{2}{|c|}{ Evenness $^{\mathrm{a}}$} \\
\hline & & & MS & $F$ & MS & $F$ & MS & $F$ & MS & $F$ \\
\hline Year & 3 & Yr*Loc(Imp) & $2.17 \times 10^{8}$ & $20.66^{* *}$ & $2.42 \times 10^{12}$ & 2.33 & $9.34 \times 10^{7}$ & $5.10 \times 10^{8 * *}$ & $9.33 \times 10^{7}$ & $1.54 \times 10^{9 * *}$ \\
\hline Impact & 3 & Loc (Imp) & $2.02 \times 10^{8}$ & $12.37 *$ & $1.26 \times 10^{13}$ & 1.66 & $1.34 \times 10^{6}$ & $936.32 * *$ & $1.34 \times 10^{6}$ & $944.49 * *$ \\
\hline Location(Imp) & 4 & Residual & $1.64 \times 10^{7}$ & 0.44 & $7.59 \times 10^{12}$ & $8.21 * *$ & $1.44 \times 10^{3}$ & $2.03 \times 10^{4 * *}$ & $1.42 \times 10^{3}$ & $4.53 \times 10^{4 * *}$ \\
\hline Yr*Imp & 9 & Yr*Loc(Imp) & $6.12 \times 10^{7}$ & $5.84 * *$ & $1.14 \times 10^{12}$ & 1.10 & 0.35 & 1.92 & 0.09 & 1.46 \\
\hline Yr*Loc(Imp) & 12 & Residual & $1.05 \times 10^{7}$ & 0.28 & $1.04 \times 10^{12}$ & 1.12 & 0.18 & $2.59 * *$ & 0.06 & $1.93 *$ \\
\hline Residual & 192 & & $3.70 \times 10^{7}$ & & $9.25 \times 10^{11}$ & & 0.07 & & 0.03 & \\
\hline \multirow[t]{2}{*}{ Source } & \multirow[t]{2}{*}{$\mathrm{df}$} & \multirow[t]{2}{*}{$F$-ratio vs } & \multicolumn{2}{|c|}{$\begin{array}{l}\text { Chlorophyta }^{\mathrm{a}} \\
\text { (\% cover) }\end{array}$} & \multicolumn{2}{|c|}{ Barnacles $^{\mathrm{a}}$} & \multicolumn{2}{|c|}{ P.mimula ${ }^{\mathrm{a}}$} & \multicolumn{2}{|c|}{ 'Other’ Limpets ${ }^{\mathrm{a}}$} \\
\hline & & & MS & $F$ & MS & $F$ & MS & $F$ & MS & $F$ \\
\hline Year & 3 & Yr*Loc(Imp) & $2.85 \times 10^{9}$ & $20.14^{* *}$ & $1.21 \times 10^{12}$ & 0.82 & $1.49 \times 10^{10}$ & 1.30 & $8.89 \times 10^{8}$ & 0.43 \\
\hline Impact & 3 & Loc (Imp) & $5.87 \times 10^{9}$ & $26.74 * *$ & $1.42 \times 10^{13}$ & 1.82 & $6.30 \times 10^{10}$ & 2.08 & $1.69 \times 10^{10}$ & $6.91 *$ \\
\hline Location(Imp) & 4 & Residual & $2.19 \times 10^{8}$ & $2.80^{*}$ & $7.82 \times 10^{12}$ & $9.24 * *$ & $3.07 \times 10^{10}$ & $17.81^{* *}$ & $2.45 \times 10^{9}$ & 1.41 \\
\hline Yr*Imp & 9 & Yr*Loc(Imp) & $7.10 \times 10^{8}$ & $5.02 * *$ & $9.99 \times 10^{11}$ & 0.67 & $1.19 \times 10^{10}$ & 1.04 & $2.73 \times 10^{9}$ & 1.32 \\
\hline Yr*Loc(Imp) & 12 & Residual & $1.41 \times 10^{8}$ & $1.81^{*}$ & $1.48 \times 10^{12}$ & 1.75 & $1.14 \times 10^{10}$ & $6.61^{* *}$ & $2.06 \times 10^{9}$ & 1.19 \\
\hline \multirow[t]{9}{*}{ Residual } & 192 & & $7.83 \times 10^{7}$ & & $8.46 \times 10^{11}$ & & $1.73 \times 10^{9}$ & & $1.74 \times 10^{9}$ & \\
\hline & & Source & $\mathrm{df}$ & $F$-ratio vs & \multicolumn{2}{|c|}{ Bembicium spp. $^{c}$} & \multicolumn{2}{|c|}{ S. glomerata $^{\mathrm{C}}$} & & \\
\hline & & & & & MS & $F$ & MS & $F$ & & \\
\hline & & Year & 3 & Yr*Loc(Imp) & 60.95 & $27.42 * *$ & 31.13 & $15.83^{* *}$ & & \\
\hline & & Impact & 3 & Loc (Imp) & 18.09 & 1.27 & 120.35 & $14.78^{*}$ & & \\
\hline & & Location(Imp) & 4 & Residual & 14.24 & $18.81^{* *}$ & 8.14 & $8.91 * *$ & & \\
\hline & & Yr*Imp & 9 & Yr*Loc(Imp) & 2.45 & 1.10 & 1.99 & 1.01 & & \\
\hline & & Yr*Loc(Imp) & 12 & Residual & 2.22 & $2.94 * *$ & 1.97 & $2.15^{*}$ & & \\
\hline & & Residual & 192 & & 0.76 & & 0.91 & & & \\
\hline
\end{tabular}


average rainfall was experienced (Figure 3b). These differences were largely attributable to the differences in barnacle numbers between locations in this category.

The differences in heterogeneity and evenness between locations within each impact category varied significantly between years as indicated by the significant Year x Location (Impact) interaction (Table VII, Figures 3c-d).

\subsubsection{Algae}

The significant Year x Impact interaction for Chlorophyta indicates that differences in green algal cover between impact categories changed significantly between years (Table VII, Figure 3e). While no significant differences in algal cover were measured between impact categories in 1996, algal cover in the Industry/Sewer category was significantly greater than all other categories in 1997. In 1998 and 1999 when above average rainfall was recorded, algal cover in the Industry/Sewer and Urban/Sewer categories was significantly greater than Bush and Urban categories but not significantly different to each other. Differences in Chlorophyta cover between locations varied significantly within each impact category between years as indicated by the significant Year x Location (Impact) interaction. No differences in algal cover were measured between locations in any impact category in 1996 however, significant differences were measured between Urban/Sewer locations in 1997 and between Industry/Sewer locations in 1998 and 1999.

\subsubsection{Fauna}

Density of barnacles only differed significantly between locations within the Industry/Sewer category (Figure 3f). Abundance of barnacles was significantly higher at Blackwattle Bay (IS) than at Hawthorne Canal (IS) in 1996 and 1999. 

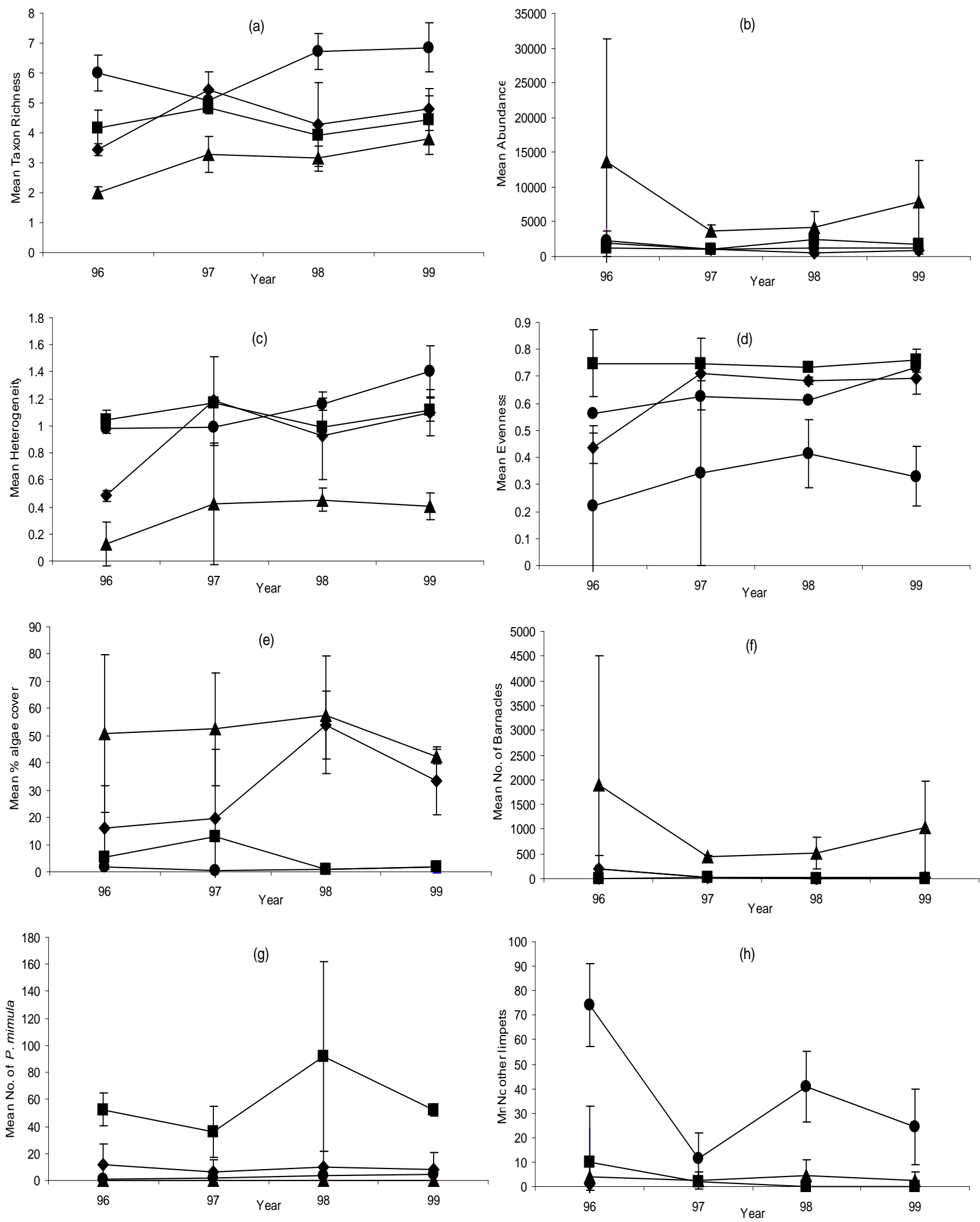

Figure 3. Means ( \pm SE; $n=2$ ) of (a) taxonomic richness, (b) total abundance, (c) heterogeneity and (d) evenness at each Sydney Harbour location in each impact category for 1996 - 1999. Means ( \pm SE; $n=2$ ) of abundance of (e) Chlorophyta, (f) barnacles, (g) Patelloida mimula and (h) 'other limpets' at each Sydney Harbour location in each impact category for 1996 - 1999. Impact categories: Industry/Sewer $(\boldsymbol{\Delta})$; Urban/Sewer ( $\bullet$ ); Urban $(\bullet)$; Bush (ø). 

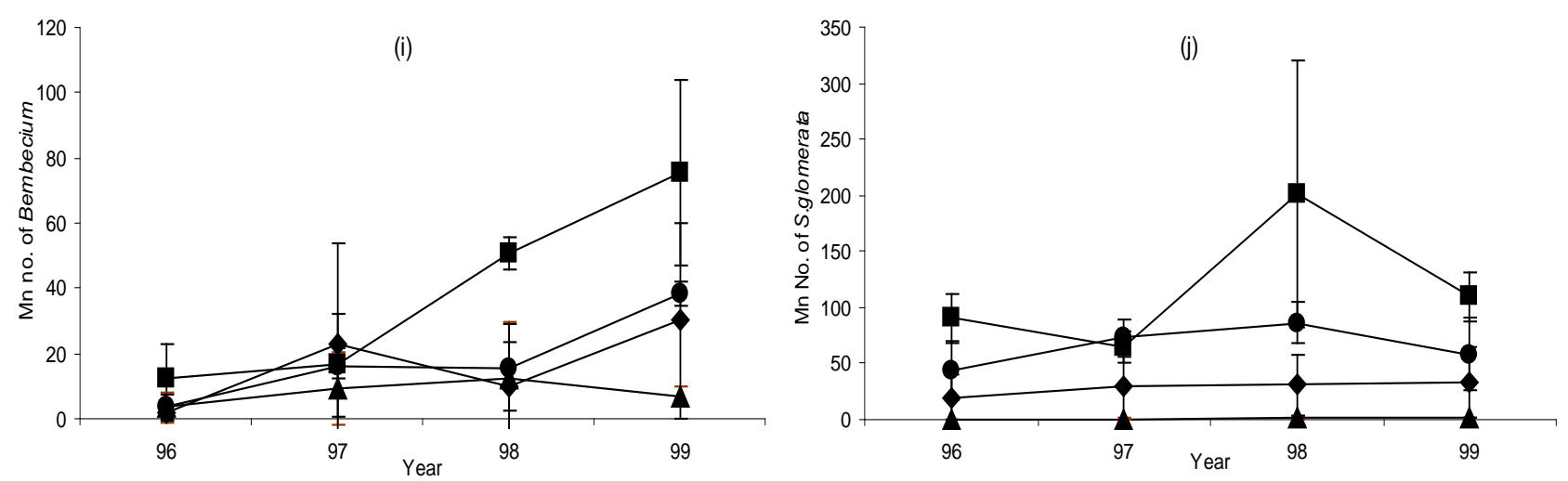

Figure 3. (continued) Means ( \pm SE; $n=2$ ) of abundance of (i) Bembecium spp. and (j) Saccostrea glomerata at each Sydney Harbour location in each impact category for 1996 - 1999. Impact categories: Industry/Sewer $(\boldsymbol{\Delta})$; Urban/Sewer $(\bullet)$; Urban (•); Bush (घ).

The significant Year x Location (Impact) interaction for density of P. mimula occurred because density varied significantly between the two Bush locations in 1998 and 1999 (Table VII, Figure 3g).

Density of 'other' limpets differed significantly between Urban and Industry/Sewer impact categories. Specifically, SNK tests identified significantly greater numbers of 'other' limpets within the Urban category than within the Industry/Sewer category (Figure 3h).

The significant Year $\mathrm{x}$ Location (Impact) interactions for Bembicium spp. and S. glomerata indicate that density of these taxa differed significantly at locations in some impact categories in some years but not all years (Table VII). SNK tests identified significant differences in density of Bembicium spp. between Bush locations in 1996, between locations within all impact categories in 1997, between locations in Industry/Sewer, Urban and Urban/Sewer locations in 1998, and between locations in Bush and Urban/Sewer locations in 1999 (Figure 3i). Density of S. glomerata varied significantly between Bush locations in 1996 and between Urban/Sewer locations in 1997, 1998 and 1999 (Figure 3j). 


\subsection{WATER QUALITY}

Water quality variables were measured at only 6, 10 and 12 of the 19 Sydney locations for biota sampling in 1995, 1996 and 1997-1999 respectively. Available data collected prior to the commencement of the biota study indicate elevated levels (relative to ANZECC guidelines) of faecal coliforms, chlorophyll-a and/or nutrients at Industry/Sewer, Urban/Sewer and Urban impact category locations, as well as at Davidson Park, a Bush location. During the study period, median levels of these variables were generally highest at the three Industry/Sewer locations and a number of Urban/Sewer locations (Cooks River, Fig Tree Bridge, Tunks Park and Rushcutters Bay). Concentrations at Davidson Park (B), however, were often similar in magnitude.

When median values for chlorophyll-a, total phosphorus and total nitrogen measured during the study were compared to low-risk guideline default trigger values applicable to New South Wales' estuaries (ANZECC, 2002), trigger guidelines were exceeded by at least one variable at all locations sampled except Gunnamatta Bay (U) and Maianbar (U). Median total phosphorus was consistently high at other locations except Little Sirius Cove (U). Median total nitrogen exceeded trigger values each year at Figtree Bridge (US) and Cooks River (US). High median chlorophyll-a values only occurred in conjunction with high total phosphorus concentrations.

\subsection{ENVIRONMENTAL INFLUENCES ON BIOTIC PATTERNS}

Chlorophyll-a was the best single correlating environmental variable in the years 1997-1999 (Table VIII). In 1996, when water quality data were not available for two of the three Industry/Sewer locations, chlorophyll-a $(\rho=0.418)$ ranked second after median conductivity $(\rho=0.481)$. In 1995, when water quality data were not available for any of the 
industry/Sewer locations, chlorophyll-a was not identified as a major contributing factor to any correlation.

TABLE VIII

Best combinations linking biotic patterns to available environmental variables.

\begin{tabular}{|c|c|c|}
\hline Period & Best single variable & Best combination of variables \\
\hline Oct 94 - Sep $95^{\mathrm{a}}$ & TUA median (0.826) & $\begin{array}{l}\text { TUA median, FC median, } \\
\text { Maximum Rainfall, Conductivity } \\
\text { median, Conductivity maximum, } \\
\text { TP median }(\mathbf{0 . 9 9 3 )}\end{array}$ \\
\hline Oct 95 - Sep 96 & Conductivity median (0.481) & $\begin{array}{l}\text { Chlorophyll-a mean, Conductivity } \\
\text { median, Total Rainfall (0.502) }\end{array}$ \\
\hline Oct 96 - Sep 97 & Chlorophyll-a median (0.758) & Chlorophyll-a median, (0.758) \\
\hline Oct 97 - Sep 98 & Chlorophyll-a mean (0.596) & $\begin{array}{l}\text { Chlorophyll-a mean, Conductivity } \\
\text { mean (0.654) }\end{array}$ \\
\hline Oct 98 - Sep 99 & Chlorophyll-a mean (0.684) & $\begin{array}{l}\text { Chlorophyll-a mean, Chlorophyll-a } \\
\text { median, Conductivity maximum } \\
(\mathbf{0 . 7 5 0 )}\end{array}$ \\
\hline
\end{tabular}

${ }^{\text {a }}$ Water quality data not available for Blackwattle Bay, Iron Cove, Little Sirius Cove, Rushcutters Bay

${ }^{\mathrm{b}}$ Water quality data not available for Iron Cove.

(Figures in bold represent Spearman rank correlation coefficients).

\section{Discussion}

Prior to the commencement of Sydney Water's major 20-year sewerage infrastructure remediation program (SewerFix) in 1997, Sydney's estuaries were receiving high pollutant loadings from stormwater runoff, sewer overflows and other diffuse sources during wet weather (AWT Ensight, 1997). Although a number of recent studies have identified changes in diversity or variability of assemblages attributable to various anthropogenic disturbances in Sydney estuaries (Kelaher et al., 1998; Glasby, 1999; Lindegarth and Hoskin, 2001), sewage and stormwater-related impact on estuarine organisms have received little attention.

The results of the present study suggest that intertidal rock assemblages are more suitable than individual taxa to assert the difference between Bush (reference) and anthropogenically disturbed estuarine locations. Although green algal cover (predominantly Enteromorpha spp. and Ulva lactuca), for example, was highest at sewage impacted locations in all years, analysis of any individual taxa was unable to reveal any differences among impact categories. 
Despite a high level of spatial and temporal variability in assemblages between locations within arbitrary impact categories, significant differences were consistently detected between the Bush category of locations, adjacent to national parks and distant from urbanisation, and those locations considered to be impacted due to their proximity to sewer overflows, and industrial and urban catchments. The differences appear to be exemplified in wet weather since Bush assemblages were significantly different to those of all other impact categories in years of above average rainfall (1998-1999). When annual rainfall was below average, Bush assemblages were not significantly different to the Urban/Sewer (in 1996-1997) or Industry/Sewer (in 1996) assemblages. Urban/Sewer assemblages and Industry/Sewer assemblages were not significantly different in any year.

Bush locations were dominated by Bembicium auratum, Saccostrea glomerata and Patelloida mimula with the latter two taxa contributing highly to differences between the Bush category and sewer impacted categories in all years. $P$. mimula were usually only found on oyster shells alongside newly settled Bembicium auratum. Furthermore, abundances of P. mimula and Bembicium spp. generally increased with numbers of oysters present. Similar associations between S. glomerata, and P. mimula and Bembicium spp. have previously been observed by Underwood and Chapman (1995) and Minchinton and Ross (1999), respectively.

In contrast, barnacles, mussels and green algae generally contributed most to similarity at Industry/Sewer locations each year. Since eutrophication usually results in reduced species diversity (Valiela, 1995), barnacles and mussels may thrive if gastropod predators such as whelks are not able to survive adverse conditions (Boaden and Seed, 1992). Increased recruitment and abundance of Mytilus edulis (one of two species of mussels recorded during this study) and Brachidontes rostratus have been observed at locations where sewage is present (Dakin, 1987; Hindell and Quinn, 2000). Furthermore, barnacles are rapid colonisers 
and may delay succession of grazers (Sutherland and Karlson, 1977; Farrell, 1991; Mann, 2000).

Reduced grazing pressure, in addition to organic enrichment, may result in colonisation by Ulva and Enteromorpha species (Underwood, 1980; Valiela, 1995; Raffaelli and Hawkins, 1999). These opportunistic algae have been associated with locations experiencing sewage discharge (Fairweather, 1990; Bellgrove et al., 1997; Panayotidis et al., 1999) and were relatively more abundant in all years at locations impacted by sewage during this study. Furthermore, cover of green algae at Urban/Sewer locations increased markedly when above average rainfall was recorded.

Hormosira banksii was not observed at any locations in Sydney Harbour. The commencement of sewage effluent discharge has been observed to greatly diminish the abundance of $H$. banksii at discharge locations (Bellgrove et al. 1997). Bellgrove et al. (1997) suggested that absence of $H$. banksii in locations experiencing environmental pressure may be a result of competitive interaction with more opportunistic species such as Ulva and Enteromorpha. In addition, inhibited germination of $H$. banksii zygotes at locations receiving sewage effluent was demonstrated by Burridge et al. (1996) with greater sensitivity of the species being displayed to poorer quality discharge. The absence of $H$. banksii throughout Sydney Harbour may be associated with sporadic contaminated freshwater input or be due to competition with other species.

Warwick and Clarke's (1993) suggestion that assemblage variability increases with anthropogenic disturbance appears to be supported by these results. Although studies by Chapman et al. (1995) and Kelaher et al. (1998) found no relationship between anthropogenic disturbance and increased assemblage variability, relative dispersion values for this study showed that variability in assemblages measured at impacted locations was consistently greater than assemblages recorded at Bush locations. Variability at the 
Urban/Sewer locations was greatest in all years sampled but particularly high in 1998 and 1999 when above average precipitation was experienced in the Sydney region.

Overlapping of replicates of different impact categories was commonly observed on nMDS ordinations. Similarity of assemblages across impact categories and differences between locations within each impact category confound the appropriateness of these impact categories. When the assemblages of all study locations are considered, it becomes apparent that some locations may have been incorrectly categorised. Since contaminants may be assimilated in different ways at different locations, the criteria for categorising impacted locations for future studies will therefore need careful consideration.

For example, assemblages at Davidson Park (B) were sampled approximately 500 metres upstream from a sewer overflow but adjacent to Garigal National Park. During 1995-1999, Davidson Park experienced relatively high levels of nutrients and chlorophyll-a yet exhibited assemblages characteristic of Bush locations. The contamination was probably due to tidal inflow transporting sewage upstream from the overflow. The contaminants would likely have been flushed with the next tidal cycle thus limiting exposure of biota to the discharge. The contaminants would likely have been flushed with the next tidal cycle thus limiting exposure of biota to the discharge. The lack of apparent impact at other locations across the greater Sydney region may possibly be due to the small size, steepness and rapid flushing of the catchment (Quakers Hat Bay) or the proximity to national parks (Sugarloaf Bay, Maianbar).

Water quality data indicated that highest contamination in Sydney Harbour occurred adjacent to stormwater canals and sewer overflows at Industry/Sewer locations (Blackwattle Bay, Hawthorne Canal and Iron Cove Creek) and a number of the Urban/Sewer locations (Fig Tree Bridge and to a lesser extent Rushcutters Bay and Tunks Park). Biological response to nutrient-enriched freshwater inflow is complex and it is difficult to draw causal links between inflow volumes, their characteristics and the resultant estuarine water quality (Chan 
and Hamilton, 2001; Pierson et al., 2002). Nevertheless, analyses of the assemblages at Industry/Sewer and Urban/Sewer locations in Sydney Harbour confirmed significant differences when compared to reference locations.

Dependent on a number of factors including residence time of contaminated water and depth of estuary, point source effluent discharges have been shown to cause increased primary production of phytoplankton, measured as chlorophyll-a concentration (Day et al., 1989; Hargrave, 1991; Valiela, 1991; Kennish, 1992; Mann, 2000; Pierson et al., 2002). This study appears to confirm that variation in chlorophyll-a is associated with variation in assemblages. Chlorophyll-a was the best single environmental variable correlating with intertidal assemblage patterns in 1997-1999. Furthermore, it is likely that the inclusion of 1995-1996 water quality data for Industry/Sewer locations would have resulted in chlorophyll-a featuring more prominently in the best combination of correlating variables in those years.

As nutrient loading increases, opportunistic pioneer flora overcome control by grazers and occupy much of the available space (Valiela, 1991; 1995). Since secondary production is ultimately dependent on primary production, sessile suspension feeders, such as barnacles in this study, flourish on decomposed phytoplankton and detrital organic matter associated with the sewage input (Boaden and Seed, 1992; Mann, 2000). Modifications to the algal community due to organic enrichment may also alter the food source for oysters and result in a decline in oyster abundance (Day et al., 1989).

\section{Summary}

Underwood (1991) suggests that an impact on biota should be deemed to have occurred when there is a significant departure in any direction from appropriate controls. It is impractical and unnecessary (and clearly difficult) to find reference locations which display identical 
characteristics. However, reference locations should be of the same general habitat and should be compared with locations in other estuaries along the coast (Underwood, 1996). Despite individual differences, the Bush locations studied in the Sydney region are relatively free of urbanisation and sewage contamination, and are arguably "the healthiest assemblage(s) that we can expect in this place(s)” (Fairweather, 1999).

In this study, significant spatial and temporal variation in assemblages was measured between locations within most impact categories in every year and between impact categories in most years. Nevertheless, variability within the Bush category assemblages was below that of the other impact categories in all years.

Although ecological responses to water quality changes within estuarine systems are difficult to predict, intertidal assemblages in Sydney Harbour and surrounding estuaries appear to be responding to the quality and quantity of discharge from urbanised catchments, particularly in years of above average rainfall. While measurement of estuarine intertidal rock assemblages appears to satisfy many of the basic criteria that an indicator should meet (Ward et al., 1998; Deeley and Paling, 1999) and provides a quantitative estimate of the environmental conditions, refinement of the impact categorisation scheme should be undertaken before future studies are conducted on a wider scale. Assuming that intertidal rock assemblages recover if a disturbance is removed, the impact of improving sewerage and stormwater infrastructure should be measurable.

\section{Acknowledgements}

Thanks to Marcus Scammell for technical input and advice, Andrew Kasmarik for his support as liaison person for Sydney Water Corporation and for arranging access to extensive water quality data, Leigh Sharp for his support as liaison person for Australian Water Technologies (AWT), and numerous AWT staff (including Paul Parkinson, Bich Pham and Sean Dwyer) for field assistance. Finally, this report is a contribution from the Centre for Sustainable Use of Coasts and Catchments, University of Newcastle. 


\section{References}

Anderson, M.J.: 2001, 'A new method for non-parametric multivariate analysis of variance', Aust. Ecol., 26, 32-46.

ANZECC: 2002, National Water Quality Management Strategy: Australian Water Quality Guidelines for Fresh and Marine Waters, Australian and New Zealand Environment and Conservation Council.

AWT - Ensight: 1996, 'Biological Indicators Pilot Studies - May 1994 to December 1995’, Prepared for Clean Waterways Programme, Sydney Water Corporation.

AWT - Ensight: 1997, 'Surveillance Monitoring Interpretive Report Port Jackson (December 1992 to January 1996)', Prepared for Clean Waterways Programme, Sydney Water Corporation. Report No: 96/086.

Axelrad, D.M., Poore, G.C.B., Arnott, G.H., Bauld, J., Brown, V., Edwards, R.R.C., and Hickman, N.J.: 1979, 'The effects of treated sewage on the biota of Port Phillip Bay, Victoria, Australia', in: B.J. Nielson and L.E. Cronin (eds), Estuaries and Nutrients, Humana Press, New Jersey, pp. 279-306.

Beck, M.W.: 1998, 'Comparison of the measurement and effects of habitat structure on gastropods in rocky intertidal and mangrove habitats', Mar. Ecol. Progr. Ser., 169, 165178.

Bellgrove, A., Clayton, M.N., and Quinn, G.P.: 1997, 'Effects of secondarily treated sewage effluent on intertidal macroalgal recruitment processes', Mar. Freshw. Res., 48, 137-46.

Bickford, G., Toll, J., Hansen, J., Baker, E., and Keesen, R.: 1999, 'Aquatic Ecological and Human Health Risk Assessment of Chemicals in Wet Weather Discharges in the Sydney Region, New South Wales, Australia', Mar. Pollut. Bull., 39, 335-45.

Boaden, P.J.S., and Seed, R.: 1992, An Introduction to Coastal Ecology, Blackie A \& P, Glasgow.

Burridge, T.R., Portelli T., and Ashton P.: 1996, 'Effect of Sewage Effluents on Germination of Three Marine Brown Algal Macrophytes’, Mar. Freshw. Res., 47, 1009-14.

Chan, T.U., and Hamilton, D.P.: 2001, 'Effect of freshwater flow on the succession and biomass of phytoplankton in a seasonal estuary’, Mar. Freshw. Res., 52, 869-84.

Chapman, M.G.: 1998, 'Relationships between spatial patterns of benthic assemblages in a mangrove forest using different levels of taxonomic resolution', Mar. Ecol. Progr. Ser., 162, 71-78.

Chapman, M.G., Underwood, A.J., and Skilliter, G.A.: 1995, 'Variability at different spatial scales between a subtidal assemblage exposed to the discharge of sewage and two control assemblages’, J. Exp. Mar. Biol. Ecol., 189, 103-122.

Clarke, K.R., and Warwick, R.M.: 2001, 'Change in Marine Communities: An Approach to Statistical Analysis and Interpretation', $2^{\text {nd }}$ ed., Plymouth Marine Laboratory, UK.

Dakin, W.J.: 1987, Australian Seashores, Angus \& Robertson, North Ryde.

Day, J.W., Hall, C.A.S., and Kemp, W.M.: 1989, Estuarine Ecology, Wiley-Interscience, New Jersey.

Deeley, D.M., and Paling, E.I.: 1999, 'Assessing the Ecological Health of Estuaries in Australia. Marine and Freshwater Research Laboratory', Land and Water Resources Research and Development Corporation Occasional Paper 17/99, Institute for Environmental Science, Murdoch University, 
Downes, B.J., and Hindell, J.S.: 2000, 'What's in a site? Variation in lotic macroinvertebrate density and diversity in a spatially replicated experiment', Aust. Ecol., 25, (2), 128-139.

Fairweather, P.G.: 1990, 'Sewage and the biota on seashores: assessment of impact in relation to natural variability’, Environ. Monitor. Assess., 14, 197-210.

Fairweather, P.G.: 1999, 'Determining the 'health' of estuaries: Priorities for ecological research', Aust. J. Ecol., 24, 441-451.

Farrell, T.M.: 1991, 'Models and mechanisms of succession: An example from a rocky intertidal community', Ecol. Monogr., 61, 95-113.

Glasby, T.M.: 1999, 'Differences Between Subtidal Epibiota on Pier Pilings and Rocky Reefs at Marinas in Sydney, Australia’, Estuarine Coastal Shelf Sci., 48, 281-290.

Hargrave, B.T.: 1991, 'Impacts of Man's Activities on Aquatic Systems', in: R.S.K. Barnes and K.H. Mann (eds), Fundamentals of Aquatic Ecology, Blackwell Science, UK, 245264.

Hindell, J.S., and Quinn, G.P.: 2000, 'Effects of sewage effluent on the population structure of Brachidontes rostratus (Mytilidae) on a temperate intertidal rocky shore', Mar. Freshw. Res., 51, 543-51.

James, R.J., Lincoln Smith, M.P., and Fairweather, P.G.: 1995, 'Sieve mesh-size and taxonomic resolution needed to describe natural spatial variation of marine macrofauna’, Mar. Ecol. Progr. Ser., 118, 187-198.

Jones, P. J., and Kaly, U. L.: 1996, 'Criteria for Selecting Marine Organisms in Biomonitoring Studies', in: R.J. Schmitt and C.W. Osenberg (eds), Detecting Ecological Impacts, Academic Press, San Diego, 29-48.

Kelaher, B.P., Chapman, M.G. and Underwood, A.J.: 1998, 'Changes in benthic assemblages near boardwalks in temperate urban mangrove forests', J. Exp. Mar. Biol. Ecol., 228, 291-307.

Kennish, M.J.: 1992, Ecology of Estuaries: Anthropogenic Effects, CRC Press, Boca Raton.

Kueh, K.S.W., and Grohmann, G.S.: 1989, 'Recovery of viruses and bacteria off Bondi Beach: a pilot study', Med. J. Aust., 151, 632-38.

Kueh, K.S.W., Grohmann, G.S., and Sheehan, D.: 1991, 'The impact of stormwater and other urban runoffs on the microbiological quality of Sydney beaches', in: Proceedings of Australian Water \& Wastewater Association Convention - Perth, 1991, Volume 2, 35664.

Lincoln Smith, M.P.: 1991, 'Environmental Impact Assessment: the Roles of Predicting and Monitoring the Extent of Impacts', Aust. J. Mar. Freshw. Res., 42, 603-14.

Lindegarth, M., and Hoskin, M.: 2001, 'Patterns of distribution of macro-fauna in different types of estuarine, soft sediment habitats adjacent to urban and non-urban areas', Estuarine Coastal Shelf Sci., 52, 237-247.

Maher, W.A., and Norris, R.H.: 1989, 'Water quality assessment programmes in Australia deciding what to measure, and how and where to use bioindicators'. Environ. Monitor. Assess., 14, 115-130.

Mann, K.H.: 2000, Ecology of Coastal Waters, Blackwell Science, UK.

Minchinton, T.E.: 1997, 'Life on the edge: conspecific attraction and recruitment of population to disturbed habitats', Oecologia, 111, pp. 45-52.

Minchinton, T.E., and Ross, P.M.: 1999, 'Oysters as habitat for limpets in a temperate mangrove forest’, Aust. J. Ecol., 24, 157-170. 
Neverauskas, V.P.: 1985, 'Effects of the Port Adelaide Treatment Works Sludge Discharge on the Adjacent Marine Environment', in: Proceedings of 1985 Australasian Conference on Coastal and Ocean Engineering, Vol. 1, 193-202.

Panayotidis, P., Feretopoulou, J., and Montesanto, B.: 1999, 'Benthic Vegetation as an Ecological Quality Descriptor in an Eastern Mediterranean Coastal Area (Kalloni Bay, Aegean Sea, Greece)', Estuarine Coastal Shelf Sci., 48, 205-214.

Pierson, W.L., Bishop, K., Van Senden, D., Horton, P.R., and Adamantidis, C.A.: 2002, 'Environmental Flows Initiative Technical Report: Environmental Water Requirements to Maintain Estuarine Processes’, Environment Australia.

Poore, G.C.B., and Kudenov, J.D.: 1978, 'Benthos around an outfall of the Werribee sewagetreatment farm', Aust. J. Mar. Freshw. Res., 29, 157-67.

Raffaelli, D., and Hawkins, S.: 1999, Intertidal Ecology, Kluwer Academic Publishers, London.

Roberts, D.E., Smith, A., Ajani, P., and Davis, A.R.: 1998, 'Rapid changes in encrusting marine assemblages exposed to anthropogenic point-source pollution: a 'Beyond BACI’ approach', Mar. Ecol. Progr. Ser., 163, 213-224.

Scammell, MS, and Collie, T.: 1994, ‘Assessment of Impact Mitigation', Australian Water Technologies discussion paper for Clean Waterways Programme, Sydney Water Board, Report No. 94/4.

Sutherland, J.P., and Karlson, R.H.: 1977, 'Development and stability of the fouling community at Beaufort, N.C.', Ecol. Monogr., 47, 425-446.

Thompson, B.W., Riddle, M.J., and Stark, J.S.: 2003, 'Cost-efficient methods for marine pollution monitoring at Casey Station, East Antarctica: the choice of sieve mesh-size and taxonomic resolution', Mar. Pollut. Bull., 46, 232-243.

Underwood, A.J.: 1980, 'The effects of grazing by gastropods and physical factors on the upper limits of distribution of intertidal macro-algae', Oceologia, 46, 201-213.

Underwood, A.J.: 1981, 'Techniques of analysis of variance in experimental marine biology and ecology', Oceanogr. Mar. Biol. Ann. Rev., 19, 513-605.

Underwood A.J.: 1991, 'Beyond BACI: Experimental designs for detecting human environmental impacts on temporal variations in natural populations', Aust. J. Mar. Freshw. Res., 42, 569-587.

Underwood A.J.: 1996, 'On Beyond BACI', in: R.J. Schmitt and C.W. Osenberg (eds), Detecting Ecological Impacts, Academic Press, San Diego, 151-175.

Underwood A.J.: 1997, Experiments in Ecology: Their Logical Design and Interpretation using Analysis of Variance, Cambridge University Press, Cambridge.

Underwood, A.J., and Chapman, M.G.: 1995, Coastal Marine Ecology of Temperate Australia, UNSW Press, Sydney.

UNESCO: 1980, 'UNESCO Technical Papers in Marine Science 1978', J. Ocean. Eng., Vol.OE-5, No.1, January 1980.

Valiela, I.: 1991, 'Ecology of Coastal Ecosystems', in: R.S.K Barnes and K.H. Mann (eds), Fundamentals of Aquatic Ecology, Blackwell Science, UK, 57-76.

Valiela, I.: 1995, Marine Ecological Processes, Springer, New York.

Vanderklift, M.A., Ward, T.J., and Jacoby, C.A.: 1996, 'Effect of reducing taxonomic resolution on ordinations to detect pollution-induced gradients in macrobenthic infaunal assemblages', Mar. Ecol. Progr. Ser., 136, 137-145. 
Ward, T.J.: 2000, 'Indicators for assessing the sustainability of Australia's marine ecosystems’, Mar. Freshw. Res., 51, 435-46.

Ward, T., Butler, E., and Hill, B.: 1998, 'Environmental Indicators for National State of the Environment Reporting: Estuaries and the Sea', CSIRO Division of Marine Research Report for Environment Australia.

Warwick, R.M. and Clarke, K.R.: 1993, 'Increased variability as a symptom of stress in marine communities’, J. Exp. Mar. Biol. Ecol., 172, 215-226. 